\title{
Figures de l'émergence de la francophonie dans les manuels scolaires pour l'Afrique et Madagascar (1960-1970)
}

Michèle Verdelhan-Bourgade

\section{(2) OpenEdition \\ Journals}

Édition électronique

URL : https://journals.openedition.org/dhfles/461

DOI : $10.4000 /$ dhfles.461

ISSN : 2221-4038

Éditeur

Société Internationale pour l'Histoire du Français Langue Étrangère ou Seconde

Édition imprimée

Date de publication : 1 janvier 2008

Pagination : 241-252

ISSN : 0992-7654

\section{Référence électronique}

Michèle Verdelhan-Bourgade, « Figures de l'émergence de la francophonie dans les manuels scolaires pour l'Afrique et Madagascar (1960-1970) », Documents pour l'histoire du français langue étrangère ou seconde [En ligne], 40/41 | 2008, mis en ligne le 14 décembre 2010, consulté le 27 mai 2021. URL : http://journals.openedition.org/dhfles/461 ; DOI : https://doi.org/10.4000/dhfles.461

Ce document a été généré automatiquement le 27 mai 2021.

(c) SIHFLES 


\title{
Figures de l'émergence de la francophonie dans les manuels scolaires pour l'Afrique et Madagascar (1960-1970)
}

\author{
Michèle Verdelhan-Bourgade
}

\section{Introduction}

1 Une réflexion sur la construction de la francophonie entre 1945 et 1970 ne peut laisser à l'écart l'enseignement du français, dans sa dimension littéraire autant que linguistique. Et le manuel à cette époque devait tenir dans cet enseignement une place importante, tant de référence culturelle que d'outil de travail. Pour rechercher l'émergence de la francophonie dans les manuels scolaires, on a eu recours à trois acceptions du terme «francophonie ». Le sens «pragmatique» du terme francophonie, en effet, change suivant le groupe qui l'utilise.

\section{Les acceptions du terme francophonie}

2 - Les politiques et les institutionnels pensent francophonie institutionnelle, à savoir l'ensemble des pays ayant le français en partage ou adhérents d'une organisation francophone. Ces pays se reconnaissent de plus comme ayant en commun des intérêts économiques, politiques et des valeurs. Leur symbole réside dans les «sommets» de la francophonie. De ce point de vue, la Roumanie, par exemple, est un pays francophone.

3 - Les linguistes parlent de francophonie linguistique, à savoir des pays dans lesquels le statut du français est celui d'une langue maternelle ou seconde. De ce point de vue, la Roumanie n'est pas un pays francophone, mais un pays où le français est langue étrangère. 
4 - Les littéraires pensent francophonie littéraire, à savoir l'ensemble des auteurs d'expression française, avec une ambiguïté de fond: on appelle souvent francophones les auteurs hors de France et non l'ensemble des auteurs de France et hors de France. C'est un débat récurrent, sans doute lié aux origines politiques de la francophonie d'après les indépendances.

5 La première question posée ici : «la francophonie existe-t-elle dans les manuels?» peut se préciser ainsi : si oui, lesquels de ces aspects sont présents dans les manuels?

\section{Le corpus}

6 On a travaillé ici sur quelques manuels utilisés pour l'enseignement du français, en Afrique francophone et à Madagascar. La liste figure en annexe. Quelques remarques.

7 - Ce sont tous des manuels parus entre 1963 et 1970, juste après les indépendances, et on peut penser que les éditeurs ont pris ce fait en considération! Après la fin de la guerre d'Algérie, c'est une période de transition avec l'ancien monde de la colonisation. Toutefois, les institutions de la francophonie ne sont pas encore créées (sauf l'AUPELF, 1961, dans le cadre multilatéral, ou la DGLF, 1966, dans un cadre d'action bilatéral, l'association des parlementaires, 1967). On est dans un entre-deux (ceci dit avec le recul): période d'après les indépendances, période d'avant l'institutionnalisation politique de la francophonie.

8 - Les manuels choisis, édités pour l'Afrique, l'ont été à partir d'une collection personnelle et des archives du CEDRHE, Centre d'études et de recherche en Histoire de l'éducation de l'IUFM de l'académie de Montpellier. Ce travail n'a aucune prétention à une quelconque exhaustivité ou même généralisation. C'est un simple constat à partir des quelques manuels examinés.

9 L'hypothèse posée était que, dans cet entre-deux, l'idée de francophonie pouvait être présente dans ces manuels de français pour l'Afrique ${ }^{1}$, sans que pour autant on la trouve sous sa forme contemporaine.

\section{La grille d'analyse}

10 Pour vérifier ou infirmer l'hypothèse, quelques indicateurs ont été retenus, notamment :

- les valeurs exaltées dans les manuels.

11 On notera au préalable qu'on ne s'attendait pas à trouver d'indication de la francophonie politique, ce qui est normal puisqu'on est dans une période antérieure à sa mise en place.

12 On n'a pas repéré non plus de présence du terme "francophonie», dans aucun des manuels étudiés. C'est peut-être moins normal, parce que le terme a quand même en 1960 quelque 80 ans d'existence. Et les préfaces, sinon le corps de manuels, auraient pu y faire référence. On regroupera ici les observations autour de trois points : la langue, la littérature et les valeurs. 


\section{Point 1. Autour de la langue française : une unité nuancée}

La langue utilisée dans les manuels est certes la langue française. Sous ce monolithisme apparent, il y a quelques modulations.

\section{Textes d'origine vs traduction}

14 Il est rarement possible de savoir si le texte utilisé est un texte véritablement francophone ou s'il est simplement traduit d'une autre langue. Or certains des auteurs convoqués n'écrivent pas en français : Gorki, Conrad, Stevenson... En dehors du manuel de $3^{\mathrm{e}}$ de la collection Écoute du monde, qui indique "traduit par... », les autres manuels ne permettent pas de faire la différence. Ce travers n'est pas spécifique aux manuels pour l'Afrique, mais très courant dans les ouvrages scolaires français de l'époque. La langue française devient donc un grand manteau qui recouvre l'ensemble des écrits littéraires, d'où qu'ils proviennent. En quelque sorte, le monde entier est francophone avant la lettre!

\section{Français vs langues nationales ou locales}

Les langues africaines ne sont pas absentes des manuels. Mais leur présence est limitée à quelques mots, souvent d'ailleurs placés en italiques (mais pas toujours). On ne trouve pas de traduction, pas non plus d'indication de la langue d'où proviennent ces mots. Parfois, au mieux, le mot est paraphrasé dans le texte lui-même :

- «faire la discrimination entre bawa, les hommes, les maîtres du tir à l'arc, et hamm'ouawa, les «femmes à moignons ", c'est-à-dire les bons à rien... »

- plus loin, dans le même texte, on aura « Attention! Attention! Mihé! Mihé! Mb'woa Gnikoni! Mb'woa Gnikoni!». (Écoute du monde, 6e, p. 225-227). Le texte est de Nazi Boni, écrivain burkinabé.

Peut-on parler d'un usage décoratif des langues africaines? Ce serait faire injure aux écrivains eux-mêmes, qui n'ont certainement pas eu cette attitude. Mais on peut le dire pour les manuels, qui, eux, ne s'intéressent pas encore réellement à ces langues ni à la position du français par rapport à elles.

\section{Un petit pas vers une francophonie linguistique}

Dans ce grand étalage tout en français, piqueté de mots en langues africaines, sans distinction d'origine, une exception notable surgit d'un texte d'André Chamson (Écoute $d u$ monde $3^{\mathrm{e}}, \mathrm{p} .336$ ). C'est une réflexion sur la place du français, à partir d'un marchandage en baoulé auquel il a assisté sur un marché de Côte d'Ivoire. Conclusion : le français appartient à ses locuteurs avec la belle formule devenue célèbre: "Une langue (...) est à ceux qui la parlent. $»^{2} C^{\prime}$ est la seule ébauche dans les manuels étudiés d'une réflexion sur la francophonie linguistique, mais elle est de taille. L'auteur va même plus loin : "Je voudrais pouvoir leur ${ }^{3}$ dire que cette langue est à eux comme ces bijoux d'or sont à moi, en toute propriété, sans réserve ni hypothèque, et qu'ils n'ont plus de compte à nous rendre de l'usage qu'ils en font. » (idem, p. 337). Bien que venant de s'extasier ici sur le raffinement mis par les locuteurs ivoiriens au maniement du 
français, Chamson affirme en fait la légitimité des variations locales du français. Le français apparaît déjà un et pluriel, ce qui n'est pas si fréquent à cette époque.

C'est donc à un début très timide de francophonie linguistique qu'on assiste, car ce texte de Chamson, le seul de l'ensemble du corpus, figure à la toute fin du manuel très copieux de classe de $3^{\text {e }}$, et il n'est pas sûr que les élèves aient l'occasion de le lire. La francophonie vue du point de vue littéraire tient-elle une place plus importante?

\section{Point 2. Francophonie littéraire : limites et ambiguïtés}

\section{Qui sont les auteurs cités ?}

19 Le tableau ci-après récapitule pour six manuels l'origine des auteurs et des textes proposés à l'étude. Quelques remarques :

20 1. Les auteurs franco-français représentent autour de $50 \%$ de la plupart des manuels. Les autres auteurs et textes d'expression française en représentent environ $25 \%$, les autres étant des textes traduits (la traduction correspond aux deux dernières lignes du tableau, en léger grisé). La proportion s'inverse pour L'école africaine qui accorde une plus grande place aux auteurs francophones.

21 2. Les pays d'où viennent les auteurs francophones sont majoritairement des pays africains d'Afrique noire, ce qui semble cohérent avec le fait que ces manuels sont utilisés pour l'Afrique. Les Antilles sont peu présentes (de 0 à 4 textes suivant les manuels), le Maghreb également (0 à 5). Le Canada est absent, la Belgique représentée deux fois par Verhaeren. Dans le manuel fait pour Madagascar, toutefois, les auteurs d'expression française sont presque exclusivement des auteurs soit malgaches, soit liés à Madagascar.

22 La part des auteurs d'expression française dans les manuels semble donc davantage liée au public visé, à l'idée que le manuel doit intéresser le public scolaire en insérant des textes d'auteurs du pays (au sens large), plutôt qu'à une idée de la francophonie, comme ensemble des écrivains de langue française. Mais l'éventail des écrivains ainsi présents est bien un début de francophonie littéraire.

\begin{tabular}{|l|l|l|l|l|l|l|}
\hline Indicateurs & $\begin{array}{l}\text { Écoute du } \\
\text { monde } \\
\text { Manuel } \\
6^{\mathrm{e}}\end{array}$ & $\begin{array}{l}\text { Écoute du } \\
\text { monde } \\
\text { Manuel } \\
5^{\mathrm{e}}\end{array}$ & $\begin{array}{l}\text { Écoute du } \\
\text { monde } \\
\text { Manuel } \\
4^{\mathrm{e}}\end{array}$ & $\begin{array}{l}\text { Écoute du } \\
\text { monde } \\
\text { Manuel } \\
3^{\mathrm{e}}\end{array}$ & $\begin{array}{l}\text { Manuel CM2 } \\
\text { L'école } \\
\text { africaine }\end{array}$ & $\begin{array}{l}\text { Manuel Mon livre } \\
\text { de français CM- } \\
\text { entrée 6e }\end{array}$ \\
\hline Nombre de textes & 109 & 98 & 101 & 183 & 81 & 87 \\
\hline Auteurs français & 49 & 54 & 57 & 89 & 19 & 39 \\
\hline $\begin{array}{l}\text { Autres auteurs } \\
\text { d'expression } \\
\text { française }\end{array}$ & 23 & 19 & 26 & 64 & 44 & 27 \\
\hline $\begin{array}{l}\text { Textes } \\
\text { francophonie }\end{array}$ & 6 & 2 & 4 & 3 & 15 & 14 \\
\hline
\end{tabular}




\begin{tabular}{|l|l|l|l|l|l|l|}
\hline $\begin{array}{l}\text { Auteurs non } \\
\text { francophones }\end{array}$ & 24 & 17 & 10 & 18 & 3 & 7 \\
\hline $\begin{array}{l}\text { Textes extérieurs à } \\
\text { la francophonie }\end{array}$ & 7 & 6 & 4 & 7 & & \\
\hline
\end{tabular}

\section{Les ambiguïtés et limites}

\section{$1^{\mathrm{er}}$ problème. Les auteurs sont rarement situés}

Il est très rare que le pays d'origine de l'auteur soit mentionné (on n'indique jamais : écrivain sénégalais, camerounais). C'est quelquefois dans le texte qu'on peut trouver des indications sur la nationalité de l'auteur (Dakar), voire dans la forme du nom, ou encore dans l'indication de l'éditeur (Yaoundé, Douala). Mais ces supputations peuvent bien sûr être fausses. L'élève n'a pas le moyen de savoir si Depestre est africain ou français (il est haïtien), ni de quel pays parle Bernard Dadié (ivoirien).

Les images sont un peu plus explicites, du moins dans la table finale des illustrations, et indiquent qu'il s'agit par exemple d'un marché ivoirien ou d'un masque du Gabon (exemple Écoute du monde, $3^{\text {e }}$, p. 378 : on ne saura pas dans le manuel que Birago Diop est sénégalais, mais on peut trouver p. 384, qu'il s'agit d'un ibis malgache). L'art est situé, la littérature non. L'ensemble des livrets Pages africaines introduit cependant à la fin de chaque tome une brève notice concernant chacun des auteurs, indiquant son pays d'origine et ses principales œuvres. Mais ces livrets ne sont pas des manuels, ce sont plutôt des compléments de type " petits classiques ", recueils de textes.

Une autre difficulté apparaît avec le manuel centré sur Madagascar. On trouve en effet indiqués sur le même plan des textes d'auteurs à noms malgaches (6), des textes d'auteurs à noms français mais qui parlent d'aspects de l'île en en montrant une connaissance précise (19), et des textes sans noms d'auteurs, indiqués. Inédits, dont on peut supposer qu'ils ont été fabriqués pour les besoins du manuel par l'un des auteurs. Les auteurs non malgaches peuvent être des Européens installés à Madagascar comme de simples touristes, on n'en saura rien. On confond ainsi auteurs du pays et auteurs qui parlent du pays, ce dernier point étant manifestement le plus important.

On a montré ailleurs (Louvain, 2005), et sur un corpus plus récent, en quoi cette façon de procéder ne contribuait pas à une éducation interculturelle. Ici, c'est l'absence de repères en matière d'écriture francophone qui frappe, ce flou des origines, qui interdit tout repérage efficace, et donc toute construction d'une vraie culture francophone.

\section{$2^{\mathrm{e}}$ problème. La représentation du monde francophone}

C'est un monde sans contours précis. Le monde francophone est peu nommé, que ce soit dans les textes ou dans les commentaires. Il peut être question de Madagascar, du Cameroun, du Dahomey ou du Tchad. Les Antilles n'existent que par rapport à l'Afrique (au travers de l'esclavage par exemple). Mais il est question aussi bien du Ghana, du lac Tanganiyka, de l'Amazonie, ou des USA. Il n'y a aucune unité francophone dans le thème du voyage. Si l'Afrique est privilégiée dans l'évocation des pays du monde, les pays francophones ne semblent pas bénéficier d'un traitement particulier. 


\section{$3^{e}$ problème. Une thématique à la fois ciblée et universaliste} stéréotypes. Mais pourquoi pas? Les stéréotypes ont bien un rôle important dans l'éducation.

36 - Des valeurs morales qu'on pourrait dire universelles: le courage, la persévérance, le travail, la camaraderie, la solidarité, s'accompagnant parfois de la dénonciation des passions (avarice, gourmandise...), l'amour du pays, etc. Toutes valeurs «traditionnelles », avec toutefois une forte insistance sur le «travail », telles qu'on pouvait les trouver dans les manuels de la première moitié du $\mathrm{XX}^{\mathrm{e}}$ siècle en France. Les auteurs de manuels ont 
privilégié dans les textes littéraires ceux qui étaient porteurs de ces valeurs-là, ce n'est pas innocent. Ce souci d'éducation morale est-il exempt de toute condescendance?

L'insistance sur ces valeurs à travers les différents textes, construit dans l'esprit du lecteur, maître ou élève, une image d'un monde, francophone, comme étant basé justement sur ces valeurs-là. Ne peut-on considérer que ce sera peut-être là un des ferments de la constitution de la francophonie, que le sentiment de solidarité entre pays partageant un certain nombre de positions?

- Des valeurs politiques et citoyennes. Plusieurs textes exaltent l'émotion du retour au pays natal, au village (que ce soit en Afrique ou à Madagascar). D'autres dénoncent l'esclavage, les guerres, et appellent à l'unité. Les manuels de $4^{\mathrm{e}}$ et de $3^{\mathrm{e}}$ se terminent par des textes poétiques (ce sont toujours par des poèmes que ces valeurs sont présentées, comme le fait d'ailleurs remarquer le manuel de $3^{\mathrm{e}}$ ) de Césaire ou de Senghor, sur une vision de l'Afrique comme ensemble, quasiment maternel, symbole d'un tiers-monde qui se lève.

Même si la francophonie politique institutionnelle est absente, par force, des manuels, on peut sans doute considérer ici que ces valeurs portées par les textes sont les prémices de la francophonie telle que la définira Senghor. Paix, solidarité, dignité des peuples longtemps opprimés vont se retrouver dans les futurs éloges de la francophonie comme piliers de ce monde nouveau.

\section{Conclusion}

40 C'est donc à une émergence ambiguë et confuse qu'on assiste dans ces manuels, en matière de francophonie. Elle n'est pas où on l'attendrait, dans l'indication explicite de la diversité des pays, des auteurs, des coutumes. Il n'y a pas non plus de référence claire à un monde francophone, mais une espèce de flou sur un ensemble d'auteurs qui utiliseraient le français pour parler du monde, et notamment de l'Afrique ou de Madagascar.

41 On est bien loin de la francophonie explicite et affichée de manuels récents, publiés notamment sous l'égide de l'AUF, bien loin aussi d'un enseignement raisonné et programmé en matière de richesses et de valeurs du monde francophone (cf. Référentiel, 2000).

42 Mais un certain nombre d'éléments convergent pour faire apparaître l'image encore incertaine d'une francophonie en construction: le fait que nombre des auteurs présents proviennent de pays divers «ayant le français en partage » comme on dira plus tard, le fait même de gommer les pays et les origines des écrivains, l'universalité des thèmes et des valeurs et un début d'affirmation politique, encore bien circonscrite à l'aire africaine, ou au " peuple noir » comme l'exprime Aimé Césaire ${ }^{6}$ :

Car pour me cantonner dans cette unique race (...).

Vous savez que ce n'est point par haine des autres races

Que je m'exige bêcheur de cette unique race... 


\section{BIBLIOGRAPHIE}

AUF (dir.) (2000). L'enseignement du français langue seconde. Un référentiel général d'orientations et de contenus, Paris, EDICEF-AUF.

GOUMAIIDA, Lynda (1999). Compétence socioculturelle : problèmes épistémologiques et didactiques (le cas de l'Algérie), thèse de doctorat, Montpellier III.

VERDELHAN-BOURGADE, Michèle (2005). «L'interculturalité en français langue seconde : une prise en compte malaisée », Colloque Quelle didactique de l'interculturel dans les nouveaux contextes d'enseignement-apprentissage du FLE/S ? Louvain.

\section{Liste des manuels}

\section{Manuels pour le second degré}

IPAM. (1965). À l'écoute du monde. Manuel de français, Collection André David, Paris, HachetteISTRA-EDICEF, $322 \mathrm{p}$.

- classe de $3^{e}(1970)$

\section{Manuels pour le premier degré}

DELCOURT, Pierre \& DUBOIS, Albert (1965). Mon livre de français. Cours moyen, entrée en sixième (livre unique), Paris, Nathan. Nathan-Madagascar.

L'école africaine (1965) Livre unique de lecture et de français, CM2, Nathan-Afrique.

\section{Premier et second degré}

VEZINET, Paul (et un groupe d'enseignants africains) (1963). Pages africaines, 5 livrets, Abidjan, CEDA-Hatier.

\section{NOTES}

1. Un des manuels est destiné à Madagascar, un autre est fait en Côte d'Ivoire, un troisième est destiné à l'Afrique sans plus de précision.

2. Texte tiré de La petite Odyssée, 1965 (date non indiquée dans le manuel).

3. Leur = à mes interlocuteurs africains.

4. Ces textes ne comportent pas de mention d'auteurs (contes, fabliaux...). Ils ne sont donc pas classés dans la rubrique « Auteurs ».

5. Auteurs francophones est pris ici au sens traditionnel d'auteurs d'expression française non français.

6. Extrait de Cahiers d'un retour au pays natal, in Pages africaines, livret 2, p. 4. 


\title{
RÉSUMÉS
}

Le français est resté après les indépendances la langue principale d'enseignement dans de nombreux pays d'Afrique, et des manuels ont été créés pour cela. On recherchera ici si la francophonie sous ses différents aspects apparaît dans les manuels scolaires destinés à l'enseignement du français en Afrique francophone, dans la tranche de temps située entre 1960 et 1970, après les indépendances, mais avant le développement de la francophonie institutionnelle. On observera la difficulté à distinguer textes français et textes traduits, et donc une sorte d'uniformisation de la littérature, qui laisse par ailleurs très peu de place aux langues nationales. Les auteurs francophones autres que français représentent environ un quart de l'ensemble, mais sont rarement situables dans un pays ou une époque. L'Afrique est traitée comme une entité, que ce soit au plan des thèmes abordés ou des valeurs exaltées. C'est ainsi à une émergence ambiguë et confuse qu'on assiste dans ces manuels, en matière de francophonie.

French continued to be taught as the language of state in many post-independence francophone countries. How were the civilizational and cultural underpinnings of this choice portrayed in the textbooks used for French instruction during the period 1960-1970? In particular, were there hints of what would later become institutionalized francophonie, the project dedicated to cultivating transnational cultural and ultimately political community through linguistic practice? If so, how were these linguistic ties portrayed? In the first place, it is hard to distinguish between texts written in French and those that have been translated, few of which were originally penned in African national languages. Similarly, while non-French, francophone authors wrote about a fourth of printed texts, they are rarely linked to a particular nation-state or period. This kind of presentation erases the distinctions between and among national, African, and European literary productions and thus de-territorializes the work presented. It also helps turn Africa into a single, homogenized entity, attributing common themes and shared values to "Africanness." I will argue that there are evocations of francophonie in these textbooks, but that their cultural and political implications are ambiguous.

\section{INDEX}

Mots-clés : Afrique, Francophonie, Madagascar, manuels scolaires

Keywords : Africa, Francophony, Madagascar, textbooks

\author{
AUTEUR \\ MICHĖLE VERDELHAN-BOURGADE \\ Université Paul Valéry-Montpellier III \\ DIDAXIS-DIPRALANG EA 739, France
}

